\title{
Espins Are Multifunctional Actin Cytoskeletal Regulatory Proteins in the Microvilli of Chemosensory and Mechanosensory Cells
}

\author{
Gabriella Sekerková, ${ }^{1,3}$ Lili Zheng, ${ }^{1,3}$ Patricia A. Loomis, ${ }^{1,3}$ Benjarat Changyaleket, ${ }^{1,3}$ Donna S. Whitlon, ${ }^{2,3}$ \\ Enrico Mugnaini, ${ }^{1,3}$ and James R. Bartles ${ }^{1,3}$ \\ Departments of ${ }^{1}$ Cell and Molecular Biology and ${ }^{2}$ Otolaryngology-Head and Neck Surgery, Feinberg School of Medicine, and ${ }^{3}$ Institute for Neuroscience, \\ Northwestern University, Chicago, Illinois 60611
}

\begin{abstract}
Espins are associated with the parallel actin bundles of hair cell stereocilia and are the target of mutations that cause deafness and vestibular dysfunction in mice and humans. Here, we report that espins are also concentrated in the microvilli of a number of other sensory cells: vomeronasal organ sensory neurons, solitary chemoreceptor cells, taste cells, and Merkel cells. Moreover, we show that hair cells and these other sensory cells contain novel espin isoforms that arise from a different transcriptional start site and differ significantly from other espin isoforms in their complement of ligand-binding activities and their effects on actin polymerization. The novel espin isoforms of sensory cells bundled actin filaments with high affinity in a $\mathrm{Ca}^{2+}$-resistant manner, bound actin monomer via a WASP (Wiskott-Aldrich syndrome protein) homology 2 domain, bound profilin via a single proline-rich peptide, and caused a dramatic elongation of microvillus-type parallel actin bundles in transfected epithelial cells. In addition, the novel espin isoforms of sensory cells differed from other espin isoforms in that they potently inhibited actin polymerization in vitro, did not bind the Src homology 3 domain of the adapter protein insulin receptor substrate p53, and did not bind the acidic, signaling phospholipid phosphatidylinositol 4,5bisphosphate. Thus, the espins constitute a family of multifunctional actin cytoskeletal regulatory proteins with the potential to differentially influence the organization, dimensions, dynamics, and signaling capabilities of the actin filament-rich, microvillus-type specializations that mediate sensory transduction in various mechanosensory and chemosensory cells.
\end{abstract}

Key words: vomeronasal organ; taste cell; Merkel cell; Müller cell; PIP2; SH3 domain

\section{Introduction}

Many classes of chemosensory and mechanosensory cells receive environmental stimuli via microvilli or microvillus-like processes, such as stereocilia. Prominent in the organization and function of these fingerlike cellular protrusions is the parallel actin bundle $(\mathrm{PAB})$ at their core. These PABs appear to act as supramolecular scaffolds that control the location, dimensions, and physical properties of microvilli and stereocilia (Bartles, 2000; DeRosier and Tilney, 2000) and may also contribute to sensory transduction by participating in the transport and positioning of the relevant cell surface proteins and downstream signaling components.

Although ostensibly a collection of actin filaments of uniform polarity cross-linked by actin-bundling proteins, PABs are nei-

\footnotetext{
Received April 5, 2004; revised May 5, 2004; accepted May 6, 2004.

This work was supported by National Institutes of Health Grants R01 DC004314 (J.R.B.) and R01 DC00653 (D.S.W.). We gratefully acknowledge Dr. Frank Margolis for providing olfactory marker protein antibody, Dr. Anthony Frankfurter for providing TuJ1 antibody, Dr. Caroline Rick for help with neuron isolation, Dr. Vijay Sarthy for helpful discussions, and Drs. Sadaf Naz and Thomas B. Friedman for conveying unpublished results.

Correspondence should be addressed to Dr. James R. Bartles, Department of Cell and Molecular Biology, Ward Building 11-185, Feinberg School of Medicine, Northwestern University, 303 East Chicago Avenue, Chicago, IL 60611. E-mail: j-bartles@northwestern.edu.

DOI:10.1523/JNEUROSCI.1279-04.2004

Copyright $\odot 2004$ Society for Neuroscience $\quad$ 0270-6474/04/245445-12\$15.00/0
}

ther uniform nor static. For example, the PABs of microvilli, stereocilia, and filopodia appear to be assembled by different mechanisms; their dimensions vary in regular ways according to location, cell type, or developmental stage; and they even appear to undergo actin treadmilling at different rates (DeRosier and Tilney, 2000; Loomis et al., 2003; Svitkina et al., 2003; Rzadzinska et al., 2004). Some of these differences may be attributed to the fact that different PABs contain different complements of actinbundling proteins (Bartles, 2000).

Previously, we identified a family of proteins, the espins, that are associated with the actin cytoskeleton. Identified originally as high-affinity actin-bundling proteins in the PABs of Sertoli cell junctions (Bartles et al., 1996; Chen et al., 1999), espin isoforms have also been found in the PABs of hair cell stereocilia (Zheng et al., 2000) and some brush border microvilli (Bartles et al., 1998) and in the actin filament-rich dendritic spines of cerebellar Purkinje cells (Sekerková et al., 2003). Encoded by a single gene, all known espin isoforms share a C-terminal 116 amino acid actinbundling module, which is necessary and sufficient for actinbundling activity in vitro (Bartles et al., 1998) and microvillar PAB elongation activity in transfected epithelial cells (Loomis et al., 2003). As a result of alternative transcriptional start-site selection and splicing, however, espin isoforms contain different $\mathrm{N}$-terminal peptides with distinct complements of protein-pro- 
tein interaction motifs (Bartles, 2000; Sekerková et al., 2003). Obvious espin orthologs are encoded in the genomes of vertebrates from Fugu to human but have not been detected in the genomes of bacteria, yeast, or nematodes. Espins show a reduced, albeit intriguing, sequence similarity to the forked proteins (Bartles, 2000), which are actin-associated proteins in the PABs of forming neurosensory bristles in Drosophila pupae (Tilney et al., 1998).

The localization of espins to hair cell stereocilia (Zheng et al., 2000; Loomis et al., 2003) and the demonstration that the espin gene is the target of mutations that cause deafness and vestibular dysfunction in mice and humans (Zheng et al., 2000; Naz et al., 2004) prompted a search for espins in other sensory cells. In this article, we show that espins are concentrated in the microvilli of a number of additional types of sensory cell. Moreover, we show that sensory cells contain novel espin isoforms that differ significantly from other espin isoforms in structure and in specific aspects of their biological activity.

\section{Materials and Methods}

Animals. Experiments used male or female adult Sprague Dawley rats and C57BL/6 mice (Harlan, Indianapolis, IN), adult homozygous jerker mice (Jackson Laboratories, Bar Harbor, ME), or newborn CD-1 mice (Charles River, Wilmington, MA). All experiments conformed to protocols approved by the Northwestern University Animal Care and Use Committee and followed guidelines issued by the National Institutes of Health and The Society for Neuroscience.

Immunocytochemistry. Organs dissected from anesthetized rodents after perfusion fixation with $4 \%$ formaldehyde were infiltrated with sucrose and sectioned on a cryostat $(25 \mu \mathrm{m})$. Whole nasal regions and temporal bones were decalcified (10\% EDTA in saline, $\mathrm{pH}$ 8.0) for 1-3 weeks before sucrose infiltration. Dissociated vomeronasal sensory neurons were prepared from 4-week-old rats using a brief (10-15 min) digestion with Pronase (Surmeier et al., 1995). Sections or dissociated neurons were labeled using standard immunofluorescence or ABC immunoperoxidase methods (Vector Laboratories, Burlingame, CA). Primary antibodies included affinity-purified rabbit polyclonal espin antibody, its corresponding preimmune IgG control (Sekerková et al., 2003), or the following: goat anti-olfactory marker protein (kindly supplied by Dr. Frank L. Margolis, University of Maryland School of Medicine, Baltimore, MD), mouse monoclonal anti-class III $\beta$-tubulin (TuJ1) (kindly supplied by Dr. Anthony Frankfurter, University of Virginia, Charlottesville, VA), mouse monoclonal anti-calretinin (Chemicon, Temecula, CA), rabbit anti- $\alpha$-gustducin (Santa Cruz Biotechnology, Santa Cruz, $\mathrm{CA}$ ), rabbit anti-ubiquitin C-terminal hydrolase (PGP 9.5) (Biogenesis, Kingston, $\mathrm{NH}$ ), mouse monoclonal anti-inositol 1,4,5-trisphosphate receptor III $\left(\mathrm{IP}_{3} \mathrm{R} 3\right)$ (Transduction Laboratories, Lexington, KY), or mouse monoclonal anti-vimentin (Sigma, St. Louis, MO). Before labeling with the $\mathrm{IP}_{3} \mathrm{R} 3$ antibody, sections were treated with $10 \mathrm{~mm}$ citric acid for $30 \mathrm{~min}$ at $80^{\circ} \mathrm{C}$ for antigen retrieval (Clapp et al., 2001). Alexa Fluor 488-, 594-, or 633-labeled goat anti-rabbit or anti-mouse secondary antibodies and Texas Red- or fluorescein-phalloidin were from Molecular Probes (Eugene, OR). Double labeling with primary antibodies from the same species was performed either sequentially using $\mathrm{Fab}^{\prime}$ fragments (Jackson ImmunoResearch Laboratories, West Grove, PA) or by the Zenon method (Molecular Probes) with similar results. An antibody specific to the larger espin isoforms was prepared by depleting affinitypurified antibody against the entire rat Purkinje cell espin 1 protein (Sekerková et al., 2003) by repeated absorption with cyanogen bromideactivated Sepharose 4B beads (Sigma) covalently derivatized with rat Purkinje cell espin 1 ( $\Delta \mathrm{N} 213)$ (see Results for more details).

Western blots, molecular biology, protein expression, and transfection. Espin proteins extracted from isolated retina or from specimens of crushed whole vomer or temporal bone by homogenization in $100^{\circ} \mathrm{C}$ SDS gel sample buffer were resolved in SDS gels and detected on Western blots using the ECL method (Amersham Biosciences, Arlington Heights, IL). The sequences of novel espin isoforms were inferred by DNA se- quence analysis of overlapping PCR products resulting from RT-PCR and $5^{\prime}$ rapid amplification of cDNA ends (RACE) PCR reactions conducted using RNA isolated from retina and selected espin primers in conjunction with kits and reagents purchased from Invitrogen (Carlsbad, CA). The PCR results were confirmed using RNA isolated from cochlear sensory epithelium prepared by microdissection of cochlear spirals obtained from newborn CD-1 mice after dispase (Calbiochem, La Jolla, CA) digestion of the cochlear capsule (D. S. Whitlon, unpublished observations). Constructs bearing truncation or deletion mutations were prepared using restriction sites or PCR, or both, and checked by DNA sequencing. Rat espins were expressed in bacteria with an $\mathrm{N}$-terminal $6 \times$ His tag and affinity purified under nondenaturing conditions (Sekerková et al., 2003) or as glutathione $S$-transferase (GST) fusions and bound to glutathione-Sepharose 4B (Loomis et al., 2003). Rat profilin I and profilin IIa (Loomis et al., 2003) and the Src homology 3 (SH3) domain of human insulin receptor substrate p53 (IRSp53) (Sekerková et al., 2003) were expressed as GST fusions and bound to glutathione-Sepharose beads. For examining effects on microvillar length, green fluorescent protein (GFP)-tagged espins were expressed by transient transfection in differentiated LLC-PK1-CL4 epithelial cells using the pEGFP-C2 vector and localized by fluorescence microscopy after fixation, detergent permeabilization, and double labeling for actin filaments with Texas Redphalloidin (Loomis et al., 2003). Microvillar PAB length was measured in oriented confocal z-sections (Loomis et al., 2003). In other experiments, untagged espins were expressed in undifferentiated LLC-PK1-CL4 cells using the pcDNA3 vector and then examined either by immunofluorescence after detergent permeabilization and double labeling with fluorescein-phalloidin or by Western blotting.

Biochemical assays. Actin filament bundling was assayed by sedimentation in the presence of either $1 \mathrm{~mm}$ EGTA or $20 \mu \mathrm{M} \mathrm{CaCl} \mathrm{C}_{2}$ (Bartles et al., 1998; Chen et al., 1999; Sekerková et al., 2003). The binding of G-actin to GST-espins and of espins to GST-profilins or GST human IRSp53 SH3 domain was measured in glutathione-Sepharose $4 \mathrm{~B}$ pull-down assays (Loomis et al., 2003; Sekerková et al., 2003). The similarity in molecular mass between certain espin isoforms and actin or the GST conjugates dictated that the assays be analyzed on Western blots labeled with espin antibody or actin antibody (C4 monoclonal; Chemicon). Fluorescence spectroscopy was used to measure the polymerization of rabbit skeletal muscle actin containing 5\% pyrene-actin (Cytoskeleton, Denver, CO) (Higgs et al., 1999). Briefly, $6 \mu \mathrm{m}$ G-actin in $5 \mathrm{~mm}$ Tris-HCl, $0.2 \mathrm{~mm}$ $\mathrm{CaCl}_{2}, 0.2 \mathrm{~mm}$ ATP, $0.5 \mathrm{~mm}$ dithiothreitol, $\mathrm{pH}$ 7.4, was converted to Mg-actin by the addition of 0.1 volume of (in mM): 10 EGTA, $1 \mathrm{MgCl}_{2}, 5$ Tris- $\mathrm{HCl}, \mathrm{pH}$ 8.0. Two minutes later, the specimen was diluted threefold by the addition of (in $\mathrm{mm}$ ): $100 \mathrm{KCl}, 1 \mathrm{MgCl}_{2}, 1$ EGTA, $0.2 \mathrm{ATP}, 0.5$ dithiothreitol, $1 \mathrm{NaN}_{3}, \mathrm{pH} 7.4$, containing different amounts of the espin isoforms, and fluorescence (excitation, $365 \mathrm{~nm}$; emission, $407 \mathrm{~nm}$ ) was monitored at $1 \mathrm{sec}$ intervals at room temperature in a PC1 photoncounting spectofluorometer (ISS, Champaign, IL) at the Keck Biophysics Facility (Northwestern University, Evanston, IL). The rate of nucleation was estimated as the reciprocal of the time required to reach $10 \%$ polymerization (Higgs et al., 1999). Binding to multilamellar phospholipid vesicles was assayed by sedimentation. Phosphatidylinositol lipids were from Calbiochem. Phosphatidylcholine (type XI-E) and all other phospholipids were from Sigma. Lipid stocks in chloroform were mixed at the desired molar ratio, evaporated to dryness under a nitrogen stream and house vacuum, swollen at $\sim 0.3 \mathrm{~mm}$ phospholipid in buffer containing (in $\mathrm{mm}$ ): $100 \mathrm{KCl}, 1 \mathrm{EDTA}, 20$ imidazole- $\mathrm{HCl}, 1$ dithiothreitol, $1 \mathrm{NaN}_{3}$, $\mathrm{pH} 7.4$, resuspended using a Vortex mixer, and centrifuged at 150,000 $\times$ $g$ for $30 \mathrm{~min}$. The multilamellar vesicles (pellet) were resuspended to $\sim 0.3 \mathrm{~mm}$ phospholipid and incubated with $\sim 5 \mu \mathrm{M}$ purified recombinant espin in the same buffer for $1 \mathrm{hr}$ at $37^{\circ} \mathrm{C}$. The multilamellar vesicles were collected by centrifugation at $150,000 \times g$ for $60 \mathrm{~min}$, and binding was assayed by comparing pellet and supernatant fractions in Coomassie Blue-stained SDS gels.

Digital image collection and processing. Digital microscopic images were captured using a Nikon PCM2000 confocal system (Melville, NY) or a Zeiss Axioplan 2 imaging microscope system with an Axiocam color camera (Thornwood, NJ). Western blot films and Coomassie Bluestained gels were digitized with an Epson Perfection 1640 SU scanner 


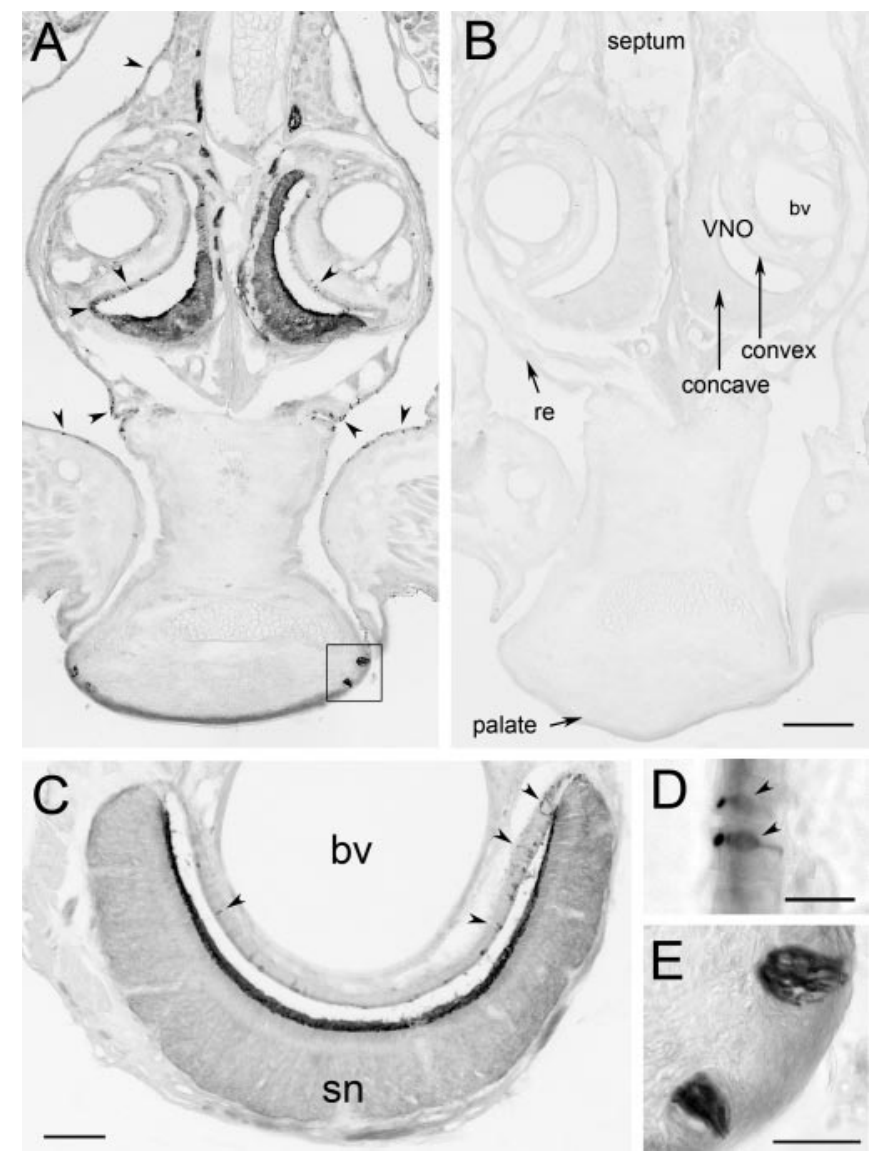

Figure 1. Immunoperoxidase localization of espins to multiple sensory structures in the oronasal cavity. $A, B$, Coronal sections through decalcified nasal region of the head from wildtype $(A)$ or homozygous jerker $(B)$ mouse. In the section from the wild-type mouse $(A)$, note the prominent reaction product in the concave aspect of the $\operatorname{VNO}(B$, see labels), in the palatal taste buds ( $A$, box in bottom right corner) and in isolated cells scattered throughout the respiratory epithelium of the nasal airways and the convex aspect of the VNO ( $A$, arrowheads). bv, Blood vessel; re, respiratory epithelium lining nasal cavity. Scale bar, $250 \mu \mathrm{m}$. C, Section through isolated mouse VNO (not decalcified). Note the prominent reaction product adjacent to VNO lumen on the concave face. sn, Sensory neurons; bv, blood vessel. Arrowheads indicate isolated espin-positive cells scattered in the epithelium on the convex aspect of the VNO. Scale bar, $50 \mu \mathrm{m}$. D, Higher-magnification view of espin-positive cells (arrowheads) scattered throughout the epithelium of the nasal cavity. Scale bar, $20 \mu \mathrm{m}$. E, Higher-magnification view of palatal taste buds from box in $A$. Scale bar, $50 \mu \mathrm{m}$.

(Epson America, Long Beach, CA). Images were processed in Photoshop 7.0 (Adobe Systems, San Jose, CA) with minor adjustments in contrast or brightness.

\section{Results}

\section{Detection of espins in multiple sensory cells}

Our search for espins in other sensory cells was initially focused on the oronasal cavity because of its richness of sensory cell types. Espin immunoreactivity was discovered in multiple sensory structures when coronal sections through the nasal region of the head were labeled with affinity-purified espin antibody (Fig. 1): the concave (sensory) face of the vomeronasal organ (VNO) (Fig. $1 A, C$ ), the palatal taste buds (Fig. $1 A, E$ ), and isolated cells scattered throughout the respiratory epithelium of the nasal airways and the convex aspect of the VNO (Fig. $1 A, C, D$, arrowheads). The staining was specific in that it was not observed when using preimmune IgG (data not shown) or when using the espin antibody to label sections from homozygous jerker mice (Fig. 1B), which lack espin protein (Zheng et al., 2000).
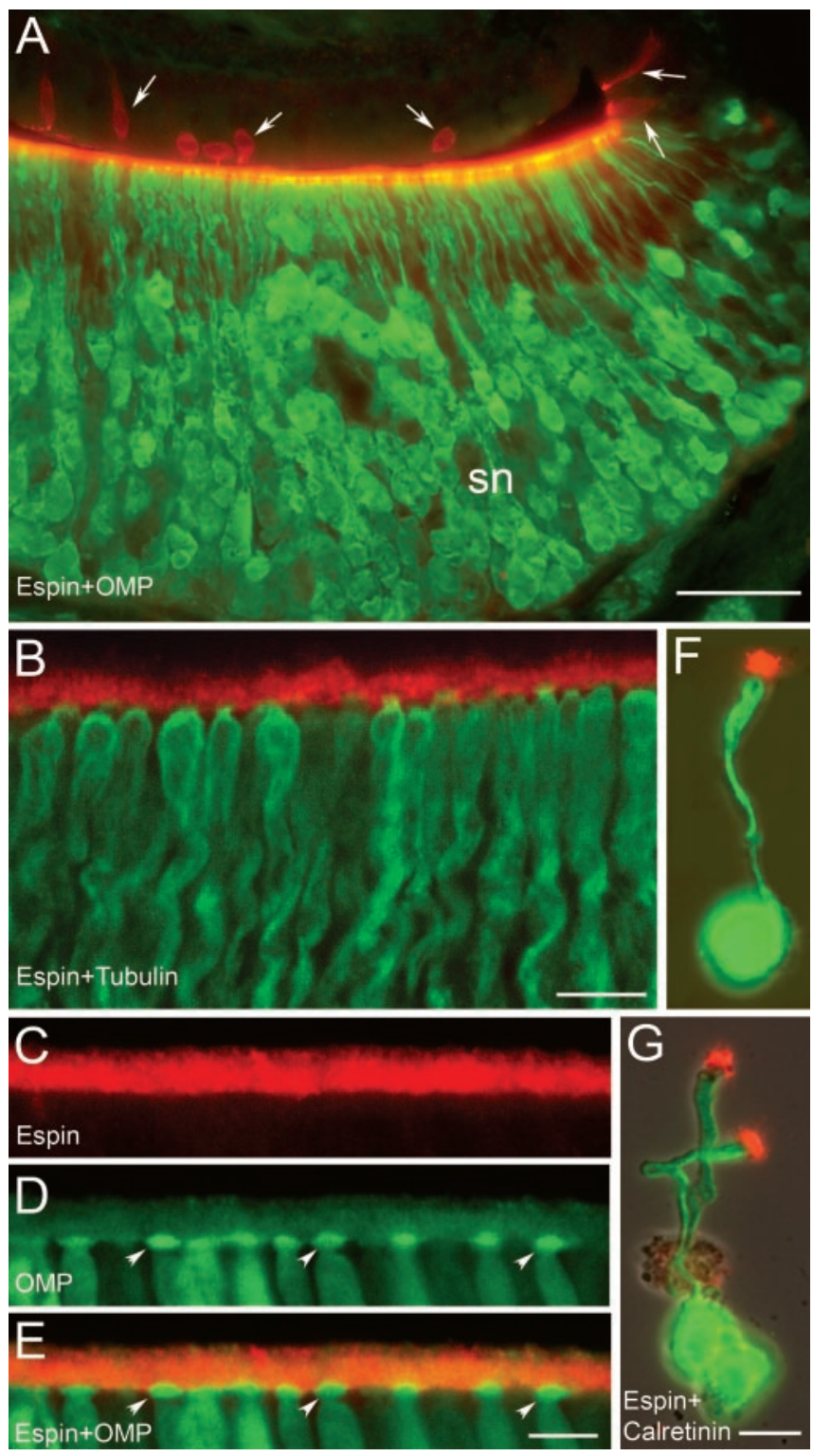

Figure 2. Immunofluorescence localization of espins to the dendritic microvilli of rat VNO sensory neurons observed in tissue sections $(A-E)$ and in isolated neurons $(F, G)$. $A$, Espin antibody (red) labels the luminal aspect of the concave face, which contains the sensory neurons (sn), as revealed by olfactory marker protein (OMP) antibody (green). The concave and convex faces are closely apposed in this section. Arrows, Isolated espin-positive cells in epithelium of convex face. Scale bar, $50 \mu \mathrm{m}$. B, Higher magnification view showing espin antibody labeling (red) of microvilli emanating from the distal ends of the neuronal dendrites, which are labeled with TuJ1 tubulin antibody (green). Scale bar, $10 \mu \mathrm{m}$. C-E, Double-labeling with espin antibody (red in $C, E$ ) and olfactory marker protein (OMP) antibody (D, E, green), which highlights the dendritic knobs ( $D, E$, arrowheads) of the VNO sensory neurons. Scale bar, $5 \mu \mathrm{m}$. F, G, Double labeling with espin antibody (red) and calretinin antibody (green) showing intense espin staining in the collection of microvilli located at the distal end of the dendrite of isolated rat VNO neurons. Scale bars, $10 \mu \mathrm{m}$.

In the $\mathrm{VNO}$, the most intense espin antibody staining was observed in a narrow zone along the luminal aspect of the concave face (Figs. $1 C, 2 A$ ). When viewed at higher magnification (Fig. $2 B-E$ ) and in isolated VNO sensory neurons (Fig. $2 F, G$ ), the source of this intense staining was identified as the dendritic microvilli that protrude from the dendritic knobs of VNO sensory neurons (Zufall et al., 2002). Espin-positive microvilli were observed on all intact isolated VNO neurons and included those 

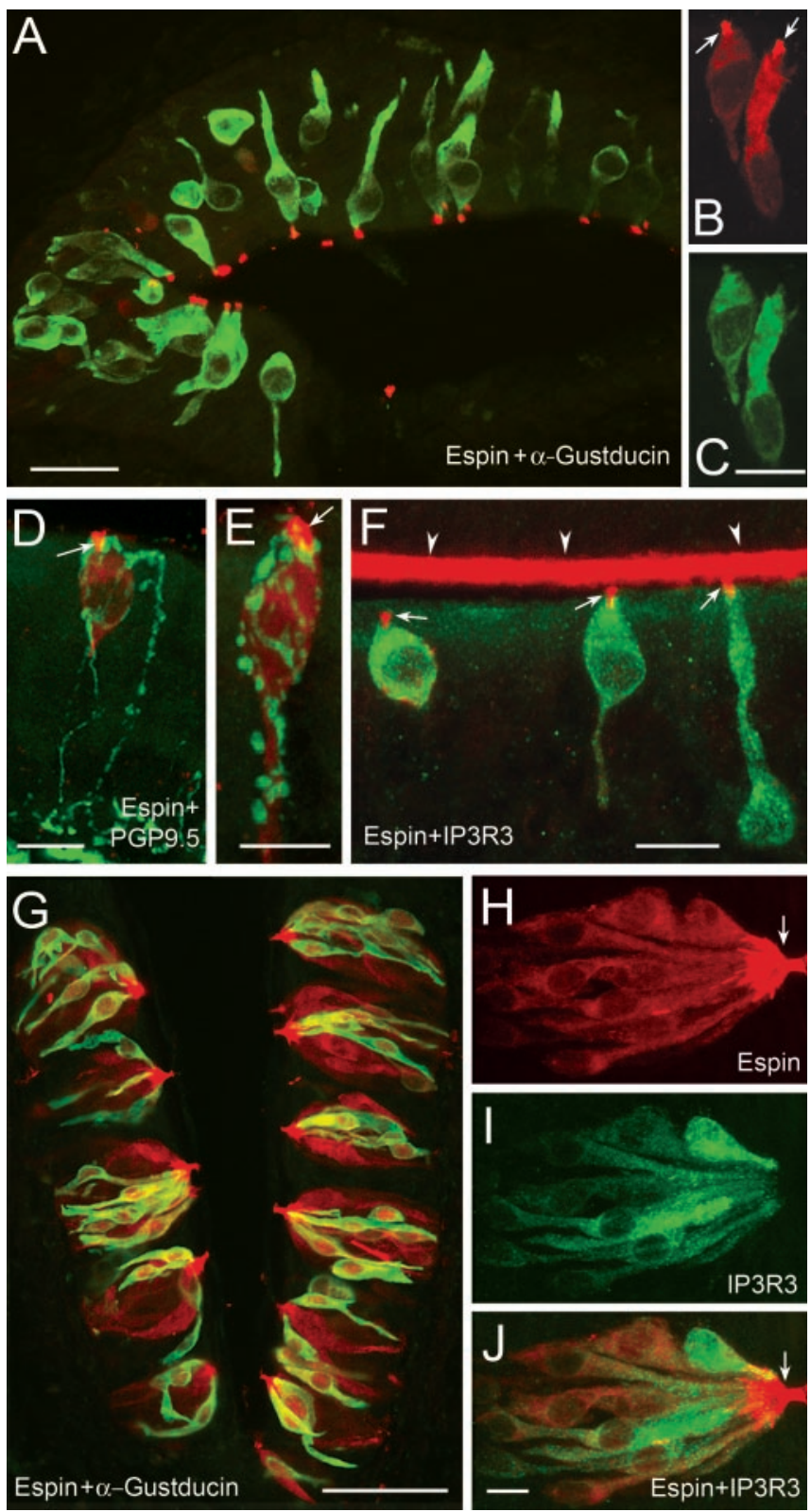

Figure 3. Immunofluorescence localization of espins to the microvilli of SCCS and taste cells. $A-F$, Sections of mouse $(A)$ or rat $(B-F)$ VN0. Espin antibody labeling (red) is concentrated in the microvilli ( $B, D-F$, arrows) of cells in the epithelium of the convex face with the properties of SCCs: the cells are labeled with $\alpha$-gustducin antibody $(A, C$, green) and in contact with nerve fibers that are labeled with PGP 9.5 antibody $\left(D, E\right.$, green). These cells are also labeled by $I_{3} R 3$ antibody $(F$, green). $F$, Arrowheads indicate espin antibody staining in the dendritic microvilli of VNO sensory neurons across the VNO lumen. Scale bars: $A, 20 \mu \mathrm{m} ;(-F, 10 \mu \mathrm{m}$. G-J, Sections of mouse $(G)$ or rat $(H-J)$ tongue showing taste buds of circumvallate papillae. Espin antibody labeling $(G, H, J$, red) is concentrated in the microvilli of taste cells $(H, J$, arrows). The espinpositive cells are labeled with $\mathbb{I}_{3} \mathrm{R} 3$ antibody $(I, J$, green), whereas fewer are labeled with $\alpha$-gustducin antibody (G, green). Scale bars: $G, 50 \mu \mathrm{m} ; J, 10 \mu \mathrm{m}$.

with short $(\sim 10 \mu \mathrm{m})$ dendrites and those with long (up to $\sim 70$ $\mu \mathrm{m})$ dendrites. This suggested that espins were concentrated in the dendritic microvilli of both apical and basal VNO sensory neurons, which bear pheromone receptors of the major families (Dulac and Torello, 2003; Fieni et al., 2003).

Opposite the dendritic microvilli across the VNO lumen, we noted a population of espin-positive cells that were distributed relatively sparsely within the epithelium covering the convex face of the VNO (Figs. $1 A, C$, arrowheads, $2 A$, arrows, $3 A-F$ ). These cells were more prevalent in the ventral and dorsal aspects of the $\mathrm{VNO}$, where the epithelium of the convex face meets that of the concave face, and were especially numerous in the anterior region of the VNO (Fig. 3A). These cells varied in shape and size and appeared highly similar to a group of espin-positive cells that we found scattered throughout the epithelium of the nasal airways (Fig. 1A,D, arrowheads). The espin antibody staining in these cells was prominent in their apical brush of stout microvilli (Fig. $3 A, B, D-F$ ) but could also be detected in the cytoplasm (Fig. $3 B, D, E)$. Whether they were located in the VNO or in the nasal cavity, these espin-positive cells were stained by antibodies to $\alpha$-gustducin (Fig. $3 A-C$ ) and therefore likely coincided with the $\alpha$-gustducin-positive cells noted previously in these locations (Zancanaro et al., 1999; Finger et al., 2003). These cells, which have been termed solitary chemoreceptor cells (SCCs), contain other components of a "bitter taste" transduction pathway and, in the nasal cavity, may trigger protective reflexes, such as apnea or sneezing, in response to irritants (Finger et al., 2003). The espin- and $\alpha$-gustducin-positive cells that we detected in the VNO and nasal cavity also resembled SCCs by the criterion that they were contacted by nerve fibers that stained with the PGP 9.5 antibody (Fig. 3D,E) (Finger et al., 2003). The SCCs in the VNO stained with antibody to $\mathrm{IP}_{3} \mathrm{R} 3$ after antigen retrieval (Fig. $3 F$ ), suggesting the presence of a phospholipase C-mediated signal transduction pathway (Clapp et al., 2004).

In addition to palatal taste buds (Fig. $1 A, E$ ), the espin antibody labeled taste buds in the fungiform, foliate, and circumvallate papillae of the tongue (Fig. $3 G, H$ ). The espin antibody appeared to stain a large percentage of the cells in taste buds (Fig. $3 H)$. Most of the espin-positive cells $(>95 \%)$ were also labeled with antibody to $\mathrm{IP}_{3} \mathrm{R} 3$ (Fig. $3 \mathrm{H}-\mathrm{J}$ ), although the ratio of $\mathrm{IP}_{3} \mathrm{R} 3$ to espin immunolabeling intensities appeared to vary among the cells within a taste bud (Fig. $3 J$, overlay). $I_{3} \mathrm{R} 3$ is a marker for a majority of type II taste cells and a subset of type III taste cells, which together are believed to be the principal transducers of bitter, sweet, and umami taste (Clapp et al., 2004). A smaller fraction of the espin-positive cells in taste buds were labeled with antibody to $\alpha$-gustducin (Fig. $3 G$ ), which is present in a fraction of the taste cells that respond to bitter stimuli (Caicedo et al., 2003). In all taste cells, the apical microvilli were labeled most intensely with the espin antibody, especially where the microvilli congregate in the taste pore (Fig. $3 G, H$ ).

The espin antibody also stained Merkel cell mechanoreceptors (Halata et al., 2003) in the basal layer of the stratified squamous epithelium of the facial epidermis (Fig. $4 A$ ) and the hard palate (Fig. $4 B$ ), as well as in association with sinus hairs (vibrissae or whiskers) (data not shown). The Merkel cells were stained with a characteristic porcupine-like pattern; their spiky microvilli, which extend into the vicinal epithelium, stained more intensely than the rest of the cell (Fig. 4A,B). The Merkel cells were innervated by nerve fibers that stained with the TuJ1 antibody to class III $\beta$-tubulin (Fig. $4 B$ ).

In retina, the espin antibody did not stain the photoreceptor cells, but instead stained Müller glial cells (Fig. 4C,D), which span virtually the entire thickness of the neural retina. The staining was most intense for the Müller cell microvilli (Fig. 4C-G), which embrace the inner segments of the photoreceptor cells, but was also detected at low levels in the thin cytoplasmic processes of the Müller cells, which were shown to better advantage by double labeling with vimentin antibody (Fig. 4D). 

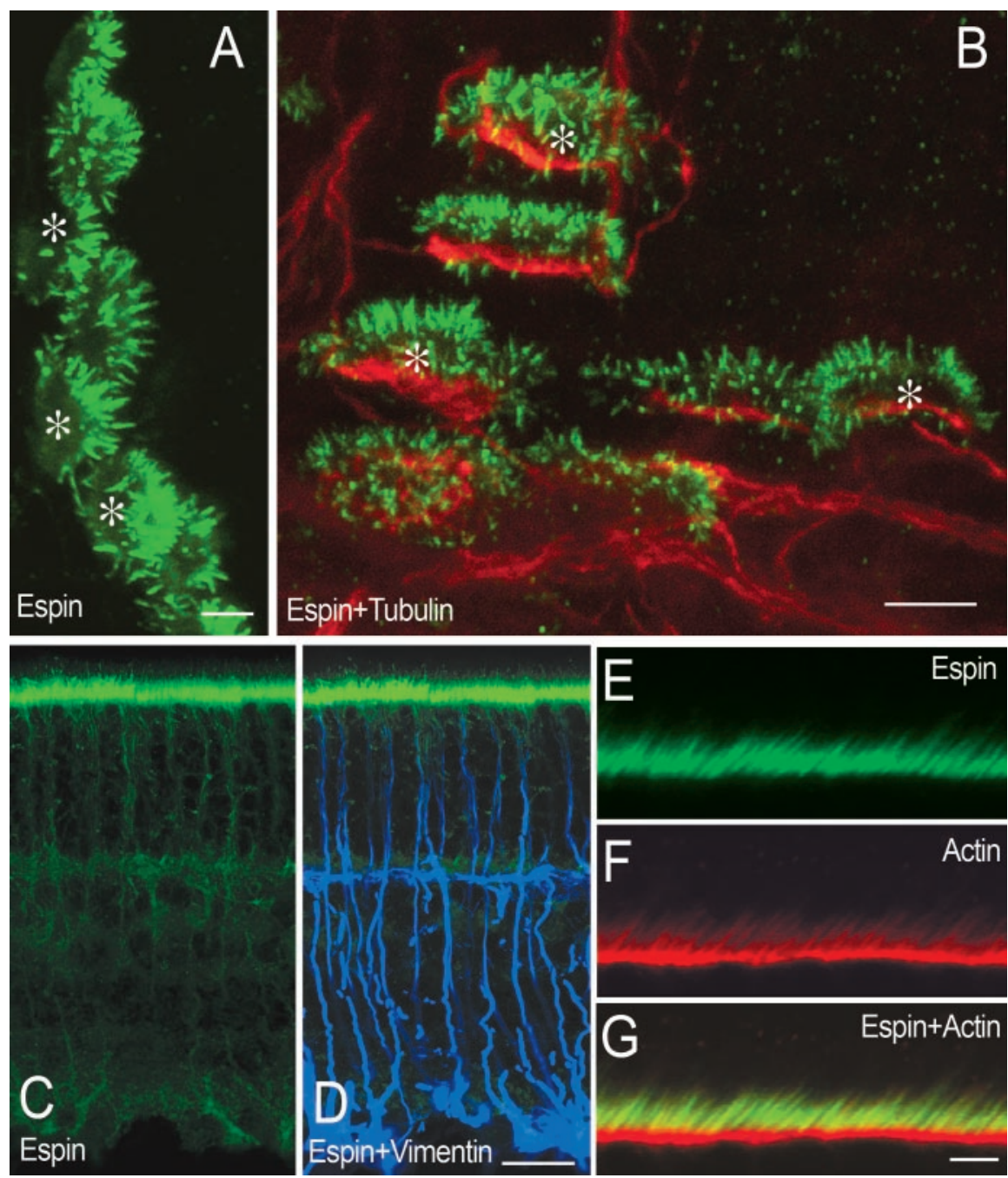

Figure 4. Immunofluorescence localization of espins to the microvilli of Merkel cells and retinal Müller glial cells. $A, B$, Sections of mouse facial skin $(A)$ and hard palate $(B)$. Espin antibody labeling (green) is concentrated in the spiky microvilli of Merkel cells (asterisks), which are located in the basal layer of the stratified squamous epithelium. The section in B is double labeled with TuJ1 tubulin antibody (red) to highlight the nerve fibers that contact the Merkel cells. Scale bars, $5 \mu \mathrm{m}$. $C-G$, Sections of rat retina. $C$, $D$, Espin antibody labeling (green) is concentrated in the microvilli of Müller glial cells but is also present at lower levels in the thin cytoplasmic processes of Müller cells, which are better revealed by double labeling with vimentin antibody $(D$, blue). Scale bar, 20 $\mu \mathrm{m} . E-G$, The espin antibody labeling of Müller cell microvilli $(E, G$, green) colocalizes with the F-actin in the PAB at their core, as revealed by double-labeling with Texas Red-phalloidin $(F, G$, red). Scale bar, $5 \mu \mathrm{m}$.

\section{Identification of novel espin isoforms in sensory cells and revision of espin nomenclature}

To identify the espin isoforms present in the sensory cells, we fractionated SDS extracts of VNO and inner ear in large-sized SDS gels, which afford a high degree of resolution, and labeled the corresponding Western blots with affinity-purified espin antibody. Both the VNO and inner ear samples contained multiple labeled bands in different molecular mass ranges (Fig. 5B). All of these bands, except a small number at low molecular mass, were labeled specifically in that they were not detected when preimmune IgG was substituted for espin antibody (Fig. 5A). Despite the apparent complexity of these Western blot patterns, it was possible to attribute most of the specifically labeled bands and multiplets to the major classes of known espin isoforms (Fig. 5B, markers at right), which can be distinguished on the basis of apparent molecular mass (Bartles et al., 1998; Sekerková et al., 2003). An exception, however, was the prominent multiplet migrating at $30-35 \mathrm{kDa}$ (Fig. $5 \mathrm{~B}$, asterisk), between the Purkinje cell espins and small espin, that was also abundant in retina (Fig. 5B). [Comparison with our published data (Bartles et al., 1996, 1998; Zheng et al., 2000; Sekerková et al., 2003) shows a systematic $5-10 \mathrm{kDa}$ decrease in apparent molecular mass of the espin isoforms on Western blots of the present specimens coincident with use of the new Invitrogen BenchMark Prestained Protein Ladder (catalog \#10748-010).]

Using a combination of RT-PCR and 5 '-RACE PCR, we identified cDNAs encoding two novel espin isoforms in RNA isolated from cochlear sensory epithelium and retina (Fig. 5D) (GenBank accession numbers AY587568 and AY587569 for rat and AY587570 and AY587571 for mouse). These two new isoforms, which differed from other known espin isoforms in transcriptional start site and splicing, were named espin $3 \mathrm{~A}$ and espin $3 \mathrm{~B}$ to reflect a revised espin nomenclature (Fig. 5D). In this new system, Arabic numerals designate espin isoforms according to transcriptional start site, and splicing variants are further specified alphabetically: Sertoli cell espin becomes espin 1, the major isoforms in cerebellar Purkinje cells become espin $2 \mathrm{~A}$ and espin $2 \mathrm{~B}$, and small espin becomes espin 4 (Fig. 5D). Although not much larger than espin 4 in molecular mass, espins $3 \mathrm{~A}$ and $3 \mathrm{~B}$ proved to be more closely related in primary structure to espin 1 and the espin 2 isoforms. For example, their coding region, which began within exon $p$, included exon $r$, but not exons $t$ and $v$, which are specific to espin 4; however, because of the selection of an alternative transcriptional start site, espin $3 \mathrm{~A}$ and espin $3 \mathrm{~B}$ have truncated $\mathrm{N}$ termini and are missing the peptides encoded by exons upstream of exon $p$, including the proline-rich peptide and additional F-actin-binding site encoded by exon $\mathrm{n}$ (Fig. 5D). In addition, as a result of differential splicing, espin $3 \mathrm{~B}$ contains a unique 30 amino acid peptide in its $\mathrm{N}$ terminus that is encoded by exon $\mathrm{q}$ (Fig. $5 D$, asterisk). The proteins expressed by the espin $3 \mathrm{~A}$ and espin $3 \mathrm{~B}$ cDNAs in transfected mammalian epithelial cells were observed to comigrate with the 30-35 kDa multiplet observed on the Western blots of VNO, inner ear, and retina (Fig. 5C).

\section{Espin isoform distribution among sensory cells}

To better define the distribution of espin isoforms among the sensory cells of these complex tissues at the protein level, we prepared an antibody that reacts with the larger espin isoforms (espin 1 and the espin 2 isoforms), but not the smaller isoforms (espins 3A, 3B, and 4). This large isoform-specific (LIS) antibody was generated by depleting our affinity-purified antibody to the entire espin 2B protein (Sekerková et al., 2003) of antibodies that recognize the smaller espin isoforms. This depletion was accomplished by repeatedly absorbing the affinity-purified espin $2 \mathrm{~B}$ antibody with Sepharose beads covalently derivatized with a frag- 


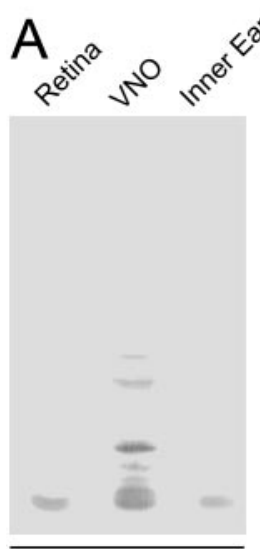

Pre-immune IgG

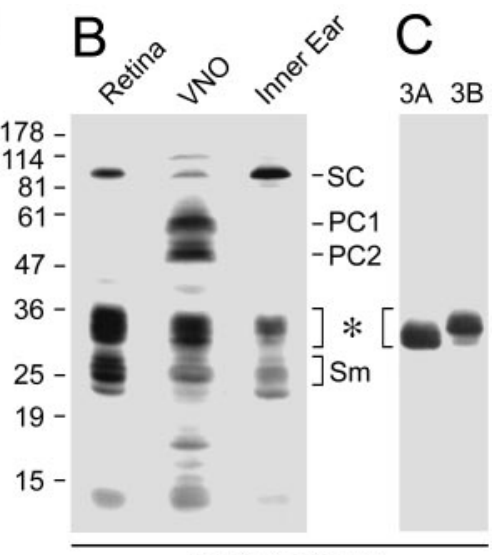

Espin Antibody
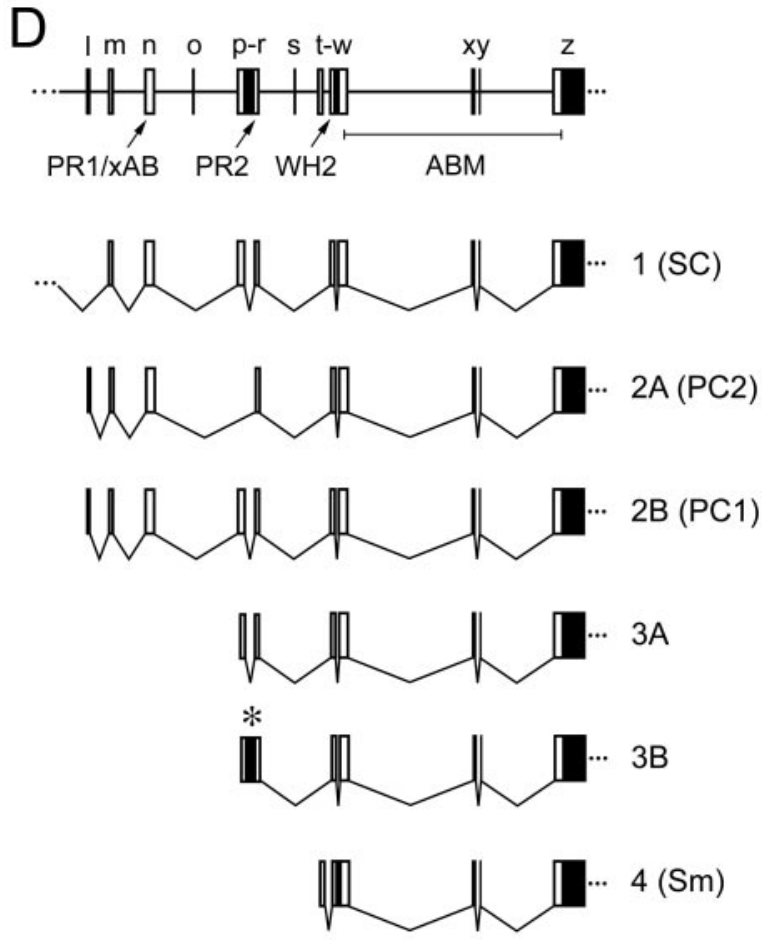

Figure 5. Western blot detection and molecular biological characterization of novel espin isoforms in sensory tissues. $A, B$, Western blots of SDS extracts prepared from the designated tissue. Note the presence of multiple bands that react with espin antibody $(B)$ but not preimmune lgG $(A)$, including a $30-35 \mathrm{kDa}$ multiplet ( $B$, bracket with asterisk) that does not correspond to any of the known espin isoforms. (This multiplet appears to run slightly slower in the retina sample, because it was close to the gel edge.) $B$, Numbers to the left, Molecular mass in kilodaltons. $B$, Abbreviations to the right, Positions of known espin isoforms (SC, Sertoli cell espin; PC1, Purkinje cell espin 1; PC2, Purkinje cell espin 2; Sm, small espin). C, Western blot showing that the newly identified espin $3 \mathrm{~A}$ and espin $3 \mathrm{~B}$ isoforms comigrate with the $30-35$ $\mathrm{kDa}$ multiplet (asterisk) when expressed in untagged form in transiently transfected LLC-PK1CL4 epithelial cells. D, Stick-figure diagrams of the structures deduced by PCR for espin $3 A$ and espin $3 B$, highlighting their utilization of espin gene exons $(I-z)$ and their relationships to the other major espin isoforms, which are designated using the new nomenclature. In parentheses are the abbreviations of the espin isoforms in the old nomenclature (see under $B$, above). The exons encoding the extended $\mathrm{N}$ terminus of espin 1 , including its eight ankyrin-like repeats, are not shown. Note that the exon-lettering scheme has also been revised (Sekerková et al., 2003) to reflect the identification of a new exon, $q$, which is unique to espin $3 B$ (asterisk). Exons $t$ and $v$ are unique to espin 4. PR1/XAB, Proline-rich peptide 1 plus additional F-actin-binding site; PR2, proline-rich peptide 2; WH2, WASP homology domain 2; ABM, actin-bundling module. ment of espin 2B that extends from the $\mathrm{C}$ terminus to just upstream of the start of the espin 3 isoforms (Fig. 5D). This absorption should leave behind only those antibodies directed against peptides encoded by exons $1-n$ and the extreme $5^{\prime}$ end of exon p, which are not included in espins 3A, 3B, or 4 (Fig. 5D).

The specificity of the LIS antibody was confirmed by immunofluorescence and by Western blotting (Fig. 6A-F). Regardless of isoform, espins elicited the formation of coarse cytoplasmic stress fiber-like F-actin bundles when expressed by transient transfection in undifferentiated LLC-PK1-CL4 epithelial cells. This caused the level of fluorescein-phalloidin staining in the espin-expressing cells to be much greater than that in neighboring, untransfected cells (Fig. 6C,E). [We have reported similar effects for espin 1,2, and 4 isoforms in transfected fibroblastic or neuronal cell lines (Bartles et al., 1998; Chen et al., 1999; Sekerková et al., 2003).] Although the LIS antibody reacted strongly with the espin 2 isoforms in the transfected cells (Fig. $6 A$ ), it did not detect the espin 3 isoforms (Fig. 6D), despite an abundance of espin 3-induced coarse cytoplasmic F-actin bundles in the transfected cells (Fig. 6E). Similarly, the LIS antibody reacted with the larger espin isoforms, but not the smaller espin isoforms, on Western blots of the purified recombinant proteins (Fig. 6F). The LIS antibody was so enriched for epitopes at the $\mathrm{N}$ terminus of espin $2 \mathrm{~B}$ that it also revealed a population of minor proteolytic breakdown products bearing $\mathrm{C}$-terminal truncations that were not evident at this exposure when labeling with the whole antibody (Fig. 6 F, right panel, weaker bands below the major espin 2A and espin 2B bands). Finally, we also titered the whole espin $2 \mathrm{~B}$ antibody by dilution to identify a concentration that yielded a staining intensity that matched that of the LIS antibody on sections of testis (Fig. $6 G, H$ ), a tissue known to contain only a large espin isoform, espin 1 (Bartles et al., 1996). Note that the staining intensities observed for the Sertoli cellspermatid junctions were closely matched between the two antibodies, and this was the case even when comparing seminiferous tubules in multiple stages of spermatogenesis (Fig. 6G,H).

The LIS antibody was then compared with the whole espin $2 \mathrm{~B}$ antibody on consecutive sections of the sensory tissues using the same dilutions that gave matched immunofluorescence intensities on sections of testis (Fig. $6 G, H$ ). Unlike the situation with the testis sections, labeling of the sensory cells with the LIS antibody resulted in either a significant reduction in staining intensity or no detectable staining. For example, the staining of the dendritic microvilli of VNO sensory neurons (Fig. 6I,J, arrowheads) and the stereocilia of vestibular hair cells (Fig. $6 \mathrm{~N}, \mathrm{O}$ ) showed reduced, albeit significant, staining with the LIS antibody. In contrast, the microvilli of the SCCs (Fig. 6I,J, arrows in $I$ ) and the stereocilia of cochlear hair cells (Fig. $6 K-M$ ) showed no detectable staining with the LIS antibody. The loss of staining was especially apparent for inner hair cells (Fig. $6 K-M$, arrows in $K, L$ ), which were labeled intensely with the whole antibody. The results obtained using the LIS antibody for these and the other cells are summarized in Figure 6 (Table, bottom right). As predicted, the LIS antibody gave no detectable signal on sections of kidney, which contains only the smaller espin isoform, espin 4 (Bartles et al., 1998) (Fig. 6, Table). Although this method is not quantitative, these results suggested that cochlear hair cells, SCCs, and taste cells are highly enriched in the smaller espin isoforms and likely include the espin 3 isoforms, which were identified as a prominent multiplet on Western blots of inner ear (Fig. $5 B$ ), and possibly also some espin 4 . In contrast, VNO sensory neurons, vestibular hair cells, Merkel cells, and retinal Müller glial cells appeared to contain a mixture of the smaller and larger isoforms. 

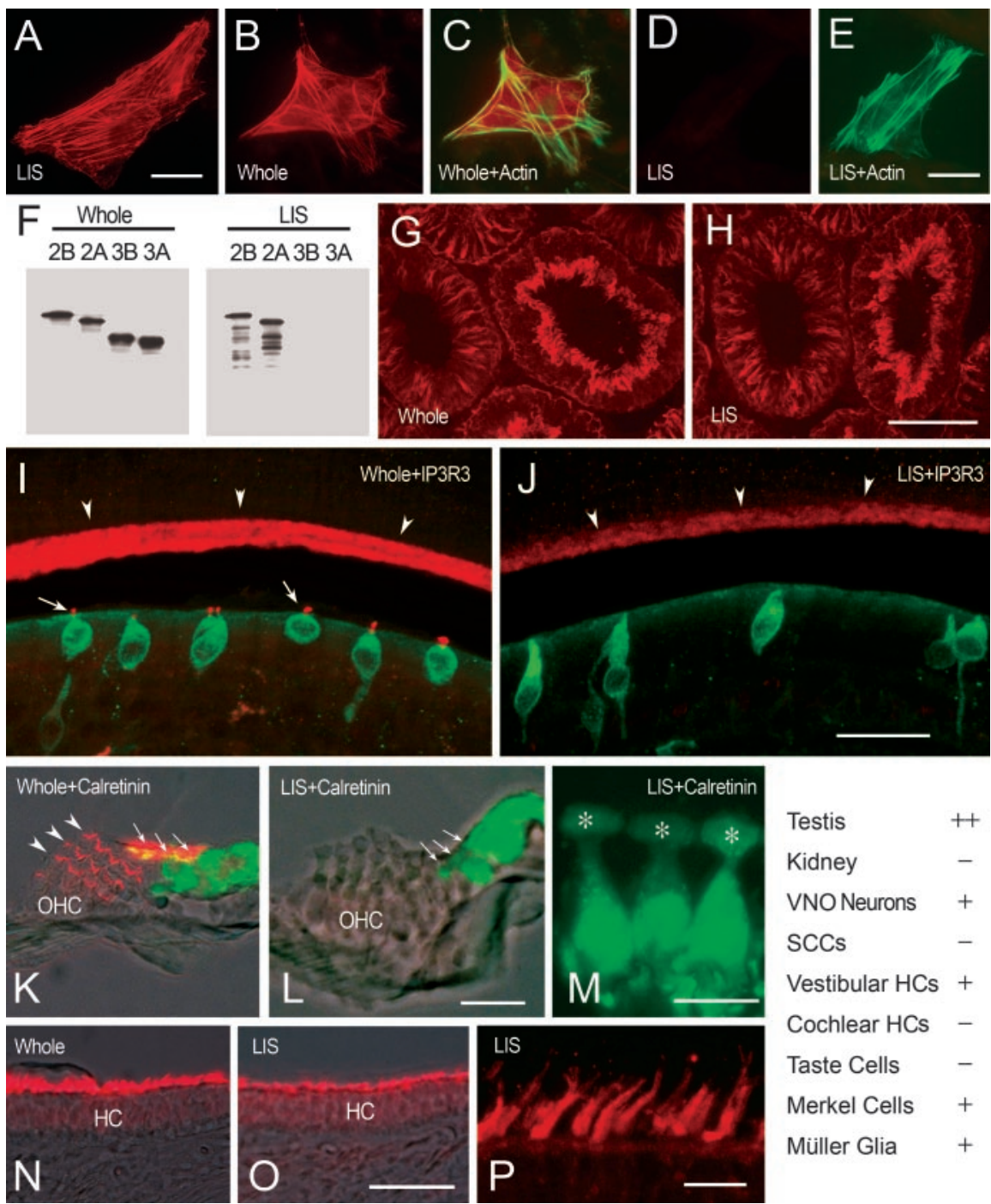

Figure 6. Espin isoform distribution among sensory cells assessed at the protein level using whole espin antibody (Whole) and the LIS espin antibody. $A-E$, Immunofluorescence of transfected cells expressing untagged espin $2 B(A)$ or espin $3 B(B-E)$ using whole ( $B, C$ or $L I S$ $(A, D, E)$ antibody (red). $C, E$, Overlays of $B$ and $D$, respectively, showing espin-induced coarse cytoplasmic $F$-actin bundles labeled with fluorescein-phalloidin (green). The LIS antibody labels espin $2 B$ in the transfected cells $(A)$, but not espin $3 B(D, E)$, despite an abundance of espin 3B-induced cytoplasmic $F$-actin bundles ( $E$ ). Scale bars, $25 \mu \mathrm{m}$. F, Identical Western blots of purified recombinant espin isoforms labeled with whole (left panel) or LIS (right panel) antibody. Note that the LIS antibody labels espins 2A and 2B, but not espins 3A and 3B, and also detects some minor, proteolytic breakdown products bearing C-terminal truncations that are not detected by whole antibody at this exposure. $G, H$, Immunofluorescence detection of espin 1 in the Sertoli cell junctions observed in consecutive sections of rat testis using diluted, whole (G), or LIS (H) antibody (red). Note that the labeling intensities obtained for the Sertoli cell junctions when using these two antibodies at these dilutions match closely, even when comparing seminiferous tubules in different stages of spermatogenesis. Scale bar, $100 \mu \mathrm{m} . I-P$, Comparisons of the immunofluorescence intensities obtained when labeling consecutive tissue sections of sensory tissues with whole or LIS antibody at the dilutions giving equal intensities on testis sections $(G, H) . I, J$, Rat VNO sections double labeled with whole ( /) or LIS ( $/$ ) antibody (red) and IP $\mathrm{P}_{3} \mathrm{R}$ antibody (green). Note reduction of labeling of neuronal dendritic microvilli (arrowheads) and loss of labeling of SCC microvilli (I, arrows) with LIS antibody ( $)$. Scale bar, $25 \mu \mathrm{m}$. K-M, Mouse cochlear sections double labeled with whole $(K)$ or LIS $(L, M)$ antibody (red) and calretinin antibody (green). Note loss of labeling in the stereocilia of inner hair cells (arrows) and outer hair cells (OHC) (K, arrowheads) with LIS antibody (L, M). M, Higher-magnification view of three inner hair cells showing absence of staining with the LIS antibody in the stereocilia, which are positioned just above the cuticular plate (asterisks). Scale bars: $L, 25 \mu \mathrm{m} ; M, 10$ $\mu \mathrm{m} . N-P$, Mouse utricular macula sections labeled with whole $(M)$ or LIS $(0, P)$ antibody (red). Note reduction in labeling of the stereocilia of vestibular hair cells $(\mathrm{HC})$ with the LIS antibody (0).P, Higher-magnification view of the collections of vestibular hair cell stereocilia labeled with LIS antibody. Scale bars: $0,50 \mu \mathrm{m} ; P, 10 \mu \mathrm{m}$. Table (bottom right) summarizes results comparing the immunofluorescence intensity obtained with the LIS antibody with that obtained with the whole espin antibody at the dilutions giving equal intensities on testis sections. ++ , No difference; + , decreased; - , not detected. HC, Hair cell.

Accordingly, in addition to the espin 3 multiplet and some espin 4, Western blots of VNO showed the espin 2 isoforms, and Western blots of inner ear showed espin 1 (Fig. $5 B$ ). We presume that the use of a harsher SDS extraction procedure accounts for why
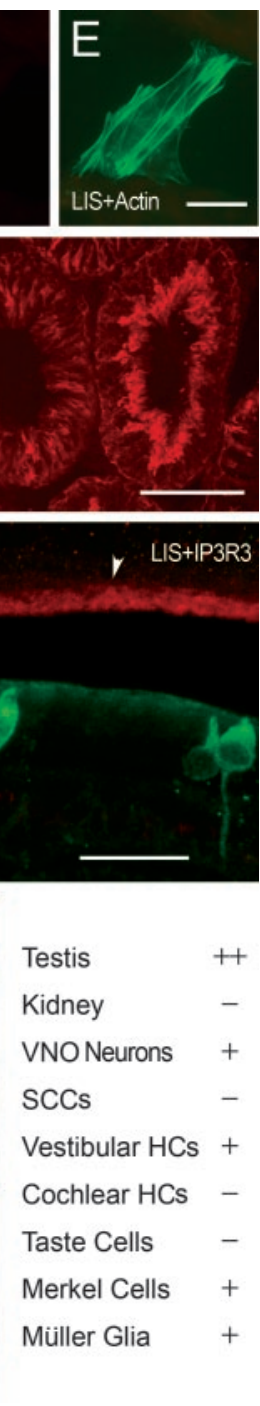

the level of espin 1 noted in rat inner ear (Fig. 5B), and apparently associated with the stereocilia of vestibular hair cells (Fig. $60, P)$, is greater than that detected previously on Western blots of extracts prepared from mouse temporal bone (Zheng et al., 2000).

\section{Biological activities of the novel espin isoforms of sensory cells}

To determine whether espin $3 \mathrm{~A}$ and espin 3B brought special biological activities to the sensory cells that contain them, we examined the properties of these novel espin isoforms in a series of biochemical and cell transfection assays. In many experiments, we compared the espin 3 isoforms with espin $2 \mathrm{~B}$ (Fig. 5D). Espin 2B, which is the major espin isoform in cerebellar Purkinje cells and differs from espin 1 by missing the eight $\mathrm{N}$-terminal ankyrin-like repeats, is the espin construct that we have characterized most extensively (Chen et al., 1999; Loomis et al., 2003; Sekerková et al., 2003). Although espins $3 \mathrm{~A}$ and $3 \mathrm{~B}$ exhibited some biological activities ascribed previously to other espin isoforms, we found that they differed from other espin isoforms in unexpected ways.

Activities dependent on the espin $C$ terminal actin-bundling module and Wiskott-Aldrich syndrome protein homology 2 domain

The two best-characterized activities of the espins are their abilities to bundle actin filaments (Bartles et al., 1998; Chen et al., 1999) and to cause the elongation of epithelial cell microvilli and their PAB scaffolds (Loomis et al., 2003). Consistent with the presence of the espin actin-bundling module in their C termini (Bartles et al., 1998; Chen et al., 1999), purified recombinant espin $3 \mathrm{~A}$ and espin $3 \mathrm{~B}$ were both highly efficient at bundling preformed actin filaments in sedimentation assays performed under physiological buffer conditions. In fact, the concentration dependence of bundling for the espin 3 isoforms was found to be similar to that measured for espin 2B (Fig. 7A). As predicted, the bundling of actin filaments by the espin 3 isoforms was not inhibited by $\mathrm{Ca}^{2+}$ (Fig. 7A, red triangle and black square at $0.04 \mathrm{~mol}$ espin $3 \mathrm{~B} / \mathrm{mol}$ actin).

The espin C-terminal actin-bundling module is also necessary and sufficient for causing the elongation of microvilli through its effects on the treadmilling actin filaments in the PAB at their core (Loomis et al., 2003). Accordingly, when expressed in differentiated LLC-PK1-CL4 epithelial cells by transient transfection, GFP-espin 3A and GFPespin $3 \mathrm{~B}$ became concentrated in microvilli and increased 
average microvillar $\mathrm{PAB}$ length from $1.33 \pm 0.04$ to $6.28 \pm 0.09$ and $6.04 \pm 0.12$ $\mu \mathrm{m}$, respectively (mean $\pm \mathrm{SE} ; n=109-$ 136 microvilli; 13-15 cells) (Fig. 7B). Although the lengthening achieved with the espin 3 isoforms was pronounced (Fig. $7 B$ ), espin $2 \mathrm{~B}$ was slightly more potent in this assay, increasing microvillar $\mathrm{PAB}$ length to $7.90 \pm 0.09 \mu \mathrm{m}$ when expressed at comparable levels (Loomis et al., 2003). Like the situation recently described for espin 2B (Loomis et al., 2003), the WiskottAldrich syndrome protein (WASP) homology 2 (WH2) consensus domain present in the espin 3 isoforms was functional in that beads loaded with GST-espin $3 \mathrm{~A}$ or GST-espin $3 \mathrm{~B}$ bound G-actin in a pull-down assay, and the binding was reduced dramatically by deletion of the WH2 domain (Fig. 7C).

Effects on actin polymerization

Despite these similarities in actin bundling, microvillar $\mathrm{PAB}$ elongation, and $\mathrm{G}$-actin binding, we found that espins $3 \mathrm{~A}$ and $3 \mathrm{~B}$ differed dramatically from espin $2 \mathrm{~B}$ in their effects on actin polymerization. In an in vitro assay that monitors actin polymerization through increases in the fluorescence of pyrene-actin tracer (Higgs et al., 1999), espin 3A and espin 3B inhibited the rate and extent of actin polymerization in a concentration-dependent manner (Fig. 7D). To our knowledge, this is the first report of an effect of an espin on actin polymerization in vitro. The inhibitory effect was pronounced even at relatively low molar ratios of espin to actin monomer (Fig. 7D). In contrast, espin 2B did not inhibit actin polymerization at these low molar ratios and, at relatively high molar ratios, actually appeared to cause modest, concentration-dependent increases in the rate and extent of actin polymerization (Fig. 7E). [The increases in pyrene-actin fluorescence observed at high molar ratios of espin 2B to actin were not attributable to actin filament bundling, because when high levels of espin $2 \mathrm{~B}$ were added to preformed actin filaments containing pyrene-actin to initiate bundling, no further increase in pyrene-actin fluorescence was observed (data not shown).] Through an analysis of the initial phase of the polymerization reaction (Higgs et al., 1999), it was possible to trace this disparity in part to an inhibitory effect of the espin 3 isoforms on the nucleation step of actin polymerization (Fig. $7 F$ ). Note that espin 4 also inhibited the nucleation of actin polymerization in this assay (Fig. $7 F$, red square).

Binding of ligands for proline-rich peptides

The espin 2 isoforms can bind IRSp53, a Cdc42- and Rac-binding adapter protein and regulator of the actin cytoskeleton (Krugmann et al., 2001; Yamagishi et al., 2004) via its SH3 domain, and this interaction is mediated specifically through the espin 2 $\mathrm{N}$-terminal proline-rich peptide (Fig. 5D, PR1) (Sekerková et al., 2003). Accordingly, when compared at binding to GST-IRSp53
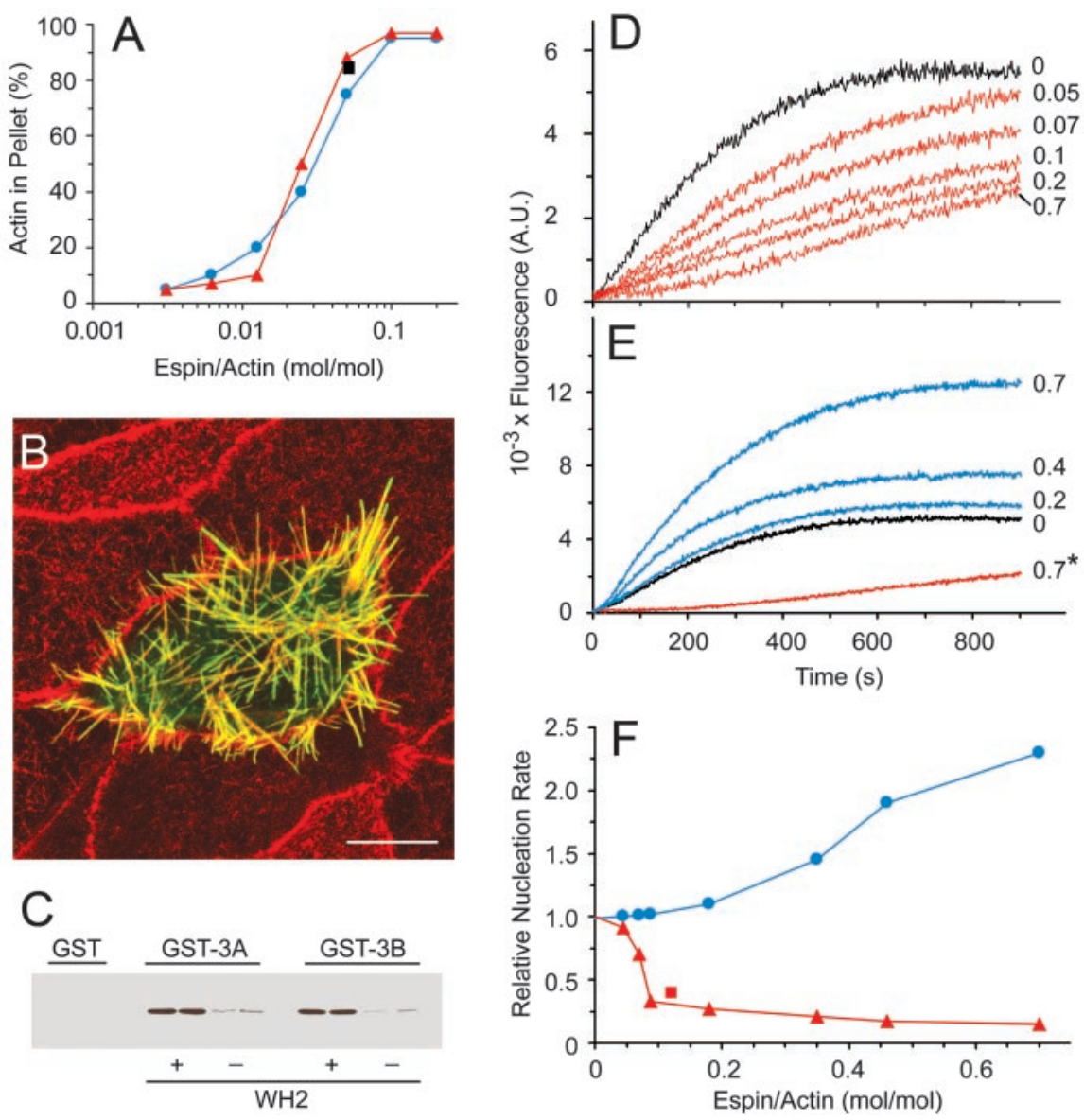

Figure 7. Interactions of the espin 3 isoforms with actin. $A$, Sedimentation actin-bundling assay. The bundling of actin filaments by espin $3 B$ (triangles, red) shows a concentration dependence similar to that for espin $2 B$ (circles, blue) and is not changed significantly when $20 \mu \mathrm{m} \mathrm{CaCl}$ (square, black) is substituted for $1 \mathrm{~mm}$ EGTA in the assay. B, Microvillar PAB elongation assay. to grow dramatically longer than those in neighboring control cells. The microvillar PABs are visualized by labeling for F-actin with exas Red-phalloidin (red). Note colocalization (yellow) of GFP-espin 3B and F-actin in the long microvilli. Scale bar, $10 \mu \mathrm{m}$. $C$ H2 domain (duplicate samples are shown). GST, Control beads loaded with GST alone. D, E, Pyrene-actin polymerization actin alone. D, Espin 3B (red curves) inhibits the polymerization of pyrene-actin in a concentration-dependent manner. E, Espin $2 B$ (blue curves) does not inhibit actin polymerization but causes a modest, concentration-dependent stimulation at high molar ratios. The inhibitory effect observed with espin $3 A$ at a molar ratio of $0.7\left(0.7^{*}\right)$ is shown for comparison (red curve). $F$, Calculated relative nucleation rates for different molar ratios of espin $3 B$ (triangles, red), espin $2 B$ (circles, blue), and espin 4 (square, red). Note that espin 3B and espin 4 inhibit nucleation, but espin 2B does not.

SH3 domain beads in a pull-down assay, espin 3A and espin 3B, which do not contain this proline-rich peptide (Fig. 5D), did not bind, whereas espin 2B bound very efficiently (Fig. $8 \mathrm{~A}$ ). The proline-rich peptides of espin $2 \mathrm{~B}$ can also bind profilins and participate in actin monomer recruitment to microvilli in vivo (Loomis et al., 2003). Profilin IIa binds proline-rich ligands with higher affinity than profilin I (Lambrechts et al., 2000). This likely explains why the efficient binding of espin 2B to GST-profilin I beads required both espin proline-rich peptides, whereas the efficient binding of espin 2B to GST-profilin IIa beads required only one proline-rich peptide (Loomis et al., 2003). In agreement with this interpretation, espin $3 \mathrm{~A}$ and espin $3 \mathrm{~B}$, which have only a single proline-rich peptide, bound efficiently to the GSTprofilin IIa beads, but at considerably lower, albeit significant, levels to the GST-profilin I beads (Fig. $8 \mathrm{~B}$ ). Consistent with the known preference of the profilins for proline-rich peptides (Pe- 


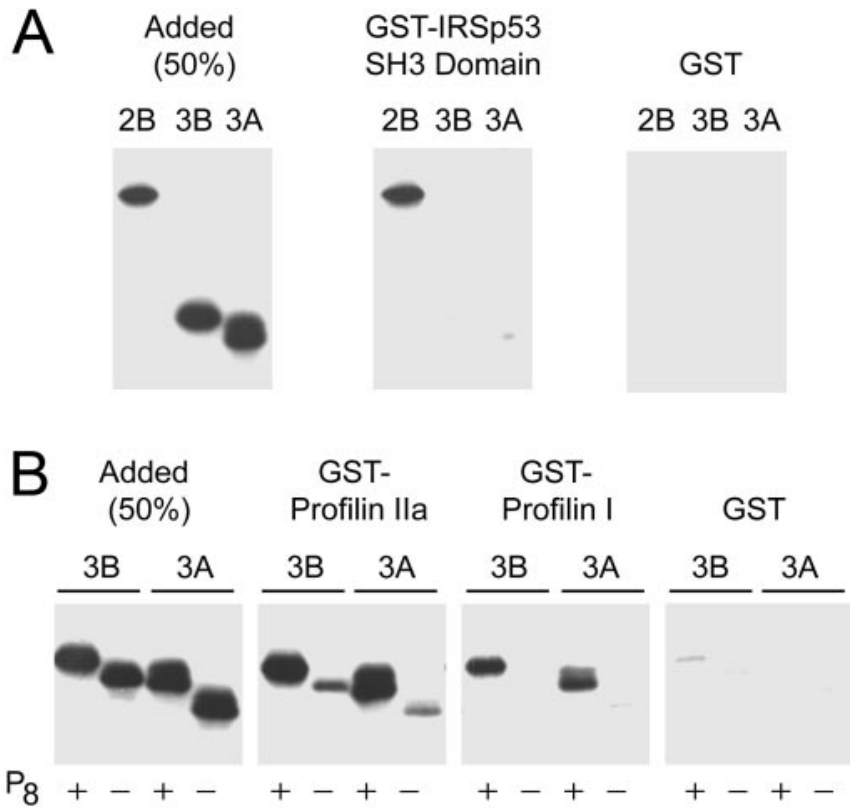

Figure 8. Interactions of the espin 3 isoforms with ligands for proline-rich peptides. A, Pulldown assay for binding the designated espin isoform to beads loaded with GST-IRSp53 SH3 domain or control beads loaded with GST. Fifty percent of the espin isoform added to the assay is shown in the left panel. Espin 2B binds to the IRSp53 SH3 domain beads, but espin 3A and espin $3 B$ do not. $B$, Pull-down assay for binding the designated espin 3 isoform, with $(+)$ or without $(-)$ the eight consecutive proline residues $\left(\mathrm{P}_{8}\right)$ in their single proline-rich peptide, to beads loaded with GST-profilin Ila, GST-profilin I, or GST. Fifty percent of the espin isoform added to the assay is shown in the left panel. The espin 3 isoforms bind better to profilin lla than profilin I, and the binding is reduced dramatically after deletion of the eight prolines.

trella et al., 1996), the binding of espin 3A and espin 3B to the GST-profilin beads was reduced dramatically after mutation to eliminate the single continuous stretch of eight proline residues from their proline-rich peptide (Fig. $8 \mathrm{~B}$ ). These results suggested that espins $3 \mathrm{~A}$ and $3 \mathrm{~B}$ contain one profilin-binding site, whereas espin 1 and the espin 2 isoforms contain two profilin-binding sites (Loomis et al., 2003).

\section{Binding to phosphatidylinositol 4,5-bisphosphate}

The acidic membrane phospholipid phosphatidylinositol 4,5bisphosphate (PIP2), which has been implicated in various sensory transduction pathways (Holy et al., 2000; Taylor, 2002; Lucas et al., 2003; Tachibana et al., 2003; Clapp et al., 2004), can mediate interactions between the actin cytoskeleton and the plasma membrane and regulate the activity or availability of a number of actin cytoskeletal proteins (Sechi and Wehland, 2000; Yin and Janmey, 2003). We compared the ability of espin isoforms to bind multilamellar phosphatidylcholine vesicles containing $20 \mathrm{~mol} \%$ of PIP2 or other phospholipids. These binding assays were performed at physiological temperature, $\mathrm{pH}$, and ionic strength; $1 \mathrm{mM}$ EDTA was included to minimize the aggregation of PIP2-containing micelles by trace divalent cations (Flanagan et al., 1997). Espin 2B and espin 4 bound efficiently to the PIP2-containing vesicles, but not to vesicles containing phosphatidylcholine alone or to phosphatidylcholine vesicles containing $20 \mathrm{~mol} \%$ of the negatively charged phospholipids phosphatidylinositol, phosphatidylserine, and phosphatidic acid (Fig. 9A). [Comparable experiments with espin 1 were not possible because recombinant espin 1 does not remain soluble under these conditions (Chen et al., 1999).] This first demonstration that certain espin isoforms can bind PIP2-containing vesicles suggested that
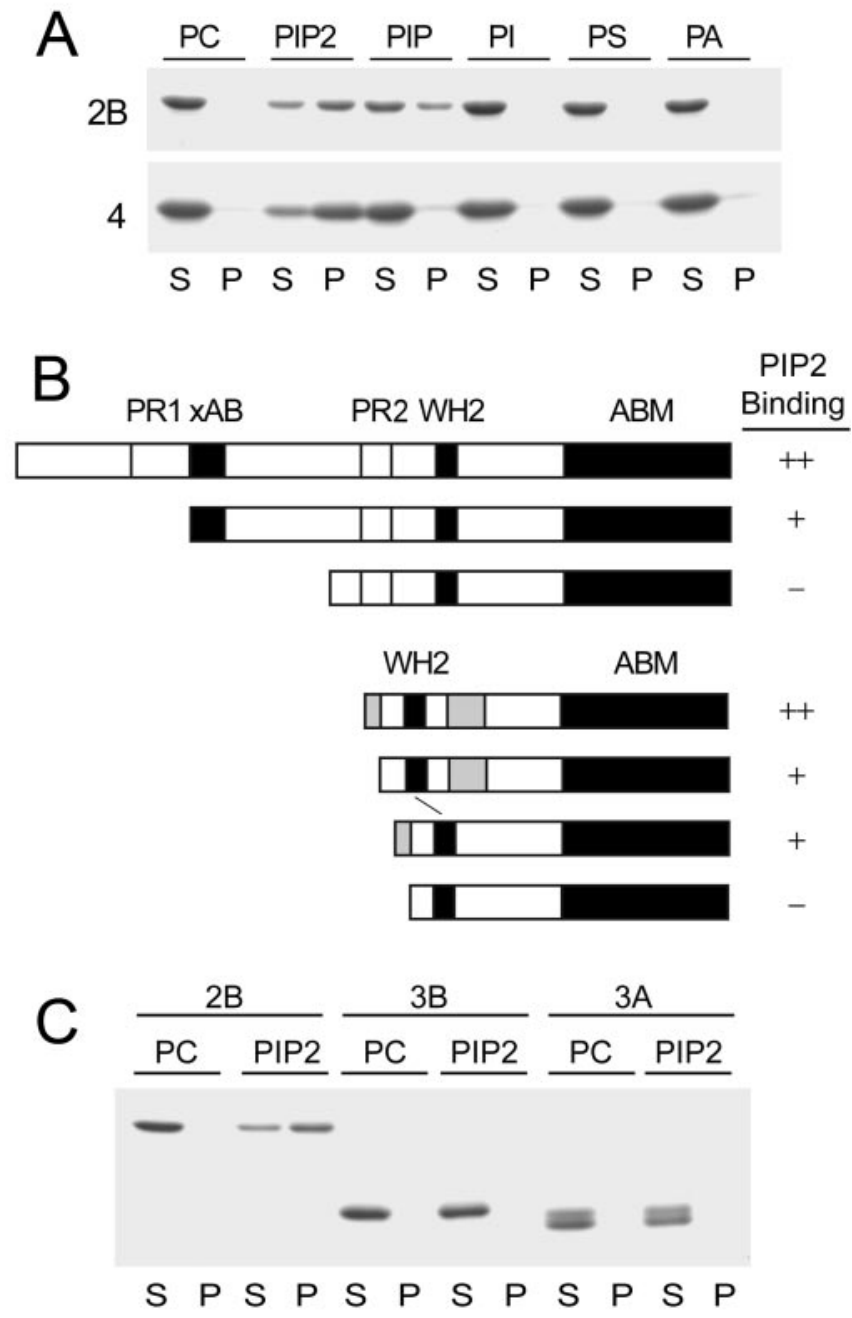

Figure 9. Interactions of espin isoforms with multilamellar vesicles containing PIP2. A, C, Coomassie Blue-stained SDS gels showing supernatant $(S)$ and pellet $(P)$ fractions obtained after incubation of the designated espin isoform with multilamellar phospholipid vesicles containing phosphatidylcholine alone (PC) or phosphatidylcholine plus $20 \mathrm{~mol} \%$ of either phosphatidyinositol 4,5-bisphosphate (PIP2), phosphatidylinositol 4-phosphate (PIP), phosphatidylinositol (PI), phosphatidylserine (PS), or phosphatidic acid (PA). Note that espin $2 \mathrm{~B}(A, C)$ and espin $4(A)$ bind specifically to vesicles containing PIP2, but espin 3A and espin $3 B$ do not $(C)$. (When purified from bacteria, espin $3 A$ often migrates as a closely spaced doublet, presumably because of partial proteolysis.) $B$, Summary of PIP2 multilamellar vesicle-binding assay results for espin 2B constructs (top three) or espin 4 constructs (bottom four) bearing truncation or deletion mutations. Note dependence on the $\mathrm{N}$-terminal part of espin $2 \mathrm{~B}$ and the unique $\mathrm{N}$-terminal peptides of espin 4 encoded by exons $t$ and $v$ (shaded gray). See legend to Figure 5 for the abbreviations used for functional domains.

these isoforms might bind to membranes or, alternatively, that they might be regulated by, or influence, signal transduction cascades (see Discussion). Espin 2B, but not espin 4, also showed significant binding to vesicles containing phosphatidylinositol 4-phosphate (Fig. 9A). Maximal binding of espin 4 to the PIP2containing vesicles required the two unique peptides in its $\mathrm{N}$ terminus (Fig. 9B, bottom four constructs). Likewise, progressive truncation of the $\mathrm{N}$ terminus of espin $2 \mathrm{~B}$ caused a stepwise reduction in the ability of espin $2 \mathrm{~B}$ to bind the PIP2-containing vesicles (Fig. 9B, top three constructs). Accordingly, espin $3 \mathrm{~A}$ and espin $3 \mathrm{~B}$, which contain neither the extended $\mathrm{N}$-terminal peptide of espin $2 \mathrm{~B}$ nor the unique peptides in the $\mathrm{N}$ terminus of espin 4 , did not bind to the PIP2-containing vesicles (Fig. 9C). 


\section{Discussion}

We have demonstrated that, in addition to the stereocilia of hair cells in the inner ear, espins are concentrated in the microvilli of a number of other types of sensory cell-VNO sensory neurons, SCCs, taste cells, and Merkel cells_-as well as retinal Müller glial cells. Moreover, in these cells we have identified novel espin isoforms that display distinct complements of ligand-binding sites and activities and therefore may impact the organization and dynamics of the actin cytoskeleton, and even signal transduction pathways, in specific ways.

The identification of espins in various sensory cells besides hair cells indicates that espins have a broader cell-type distribution than originally appreciated. The observation that espins are not present in all epithelial cells (Bartles et al., 1998; this study) or neurons (Sekerková et al., 2003) raises the question of what advantages espins bring to cells that contain them. The detection of obvious espin orthologs in vertebrates, but not in the sequenced genomes of bacteria, yeasts, or nematodes, suggests that espins are a relatively late evolutionary invention. The reduced, albeit significant, sequence similarity to the forked proteins in the developing neurosensory bristles of Drosophila (Bartles, 2000) provides an intriguing, distant evolutionary connection to cells with a sensory function.

\section{$\mathrm{Ca}^{2+}$ and phospholipase C-mediated signaling}

Unlike other actin-bundling proteins in vertebrate microvilli or stereocilia, such as fimbrin-plastin and villin, the espins bundle actin filaments efficiently even in the presence of $\mathrm{Ca}^{2+}$ (Bartles, 2000). This resistance to $\mathrm{Ca}^{2+}$ may be of special utility to sensory cells, because these cells presumably must preserve their crosslinked actin cytoskeletal structures while experiencing transient increases in $\mathrm{Ca}^{2+}$ during signal transduction via channels in the plasma membrane and release from intracellular stores. Yet, paradoxically, despite their high affinity and $\mathrm{Ca}^{2+}$ resistance, espin cross-links seem not to interfere with the actin treadmilling that is presumed to be essential for the continuous renewal of the $\mathrm{PAB}$ at the core of microvilli and stereocilia (Loomis et al., 2003; Rzadzinska et al., 2004). A role for espins in cells with $\mathrm{Ca}^{2+}$-based signaling may also explain their presence in the dendritic spines of cerebellar Purkinje cells (Sekerková et al., 2003) and retinal Müller glial cells. The dendritic spines of Purkinje cells experience some of the largest $\mathrm{Ca}^{2+}$ transients in the brain (Hansel et al., 2001), and Müller cells experience transient increases in intracellular $\mathrm{Ca}^{2+}$ that can be propagated to neighboring Müller cells (Newman, 2001).

Increases in intracellular $\mathrm{Ca}^{2+}$ often occur downstream of the receptor-mediated activation of phospholipase $\mathrm{C}$ and its cleavage of PIP2 to diacylglycerol and inositol 1,4,5-trisphosphate (Taylor, 2002). Moreover, phospholipase C-mediated signaling is emerging as a common theme among sensory cells that contain espins (Holy et al., 2000; Lucas et al., 2003; Tachibana et al., 2003; Clapp et al., 2004). Thus, it may be pertinent that certain espin isoforms can bind multilamellar phospholipid vesicles containing PIP2, whereas others cannot. Although the espin isoforms that bind PIP2 do not appear to contain recognizable phosphoinositide-binding motifs (Cullen et al., 2001), their binding to PIP2 requires $\mathrm{N}$-terminal peptides that contain clusters of amino acids with positively charged side chains. PIP2 is known to regulate the activity or availability of a number of actin cytoskeletal proteins by binding to peptides rich in positively charged amino acids (Sechi and Wehland, 2000; Yin and Janmey, 2003). Conversely, some PIP2-binding proteins can reduce the availability of PIP2 for cleavage by phospholipase C (Goldschmidt-
Clermont et al., 1990; Wang et al., 2002). Thus, the absence of PIP2binding activity that we noted for the espin 3 isoforms might prevent them from binding to membranes or interfering with phospholipase C-mediated signaling. Alternatively, the inability to bind PIP2 might provide a means to uncouple the espin 3 isoforms from regulation by polyphosphoinositides.

\section{Microvillar dimensions and sensory transduction}

We have shown previously that espins can increase the length and diameter of microvillus-type cell-surface projections via effects on their dynamic PAB scaffolds (Loomis et al., 2003). Thus, espins may control the dimensions and, hence, also the physical properties of the specializations of sensory cells that are believed to be the site of sensory transduction. In this capacity, espins may help give the microvillus-like projections that adorn each class of sensory cell their characteristic shape, which can range from the stout microvilli of the SCCs to the long, spiky microvilli of Merkel cells. In the case of hair cells, the graded regulation of stereocilium length to produce a staircase assembly is a likely prerequisite for effective mechanosensory transduction (Pickles and Corey, 1992). It is even possible that the gradient in stereocilium length observed along the cochlear spiral (Kaltenbach et al., 1994), which we have shown to be correlated with espin level (Loomis et al., 2003), could contribute to tonotopic frequency mapping (Ricci et al., 2003). Accordingly, many deafness mutations, including the jerker mutation in the espin gene (Zheng et al., 2000), cause aberrations in stereocilium length, width, or organization (Karolyi et al., 2003). Although it is not difficult to envision how the number and length of microvilli could affect the mechanosensory function of Merkel cells (Halata et al., 2003), experiments examining the role of microvillar dimensions in the signaling of Merkel cells or most other sensory cells are lacking.

\section{Actin monomer recruitment and polymerization}

Beyond actin bundling and microvillar PAB elongation, the espins display a number of additional binding sites and activities that could regulate the organization and dynamics of the actin cytoskeleton in sensory cells. For example, all known espin isoforms contain a WH2 actin monomer-binding domain. Best characterized for the WASP family of nucleation-promoting factors involved in Arp2/3-mediated actin polymerization (Welch and Mullins, 2002), the WH2 domain is also present in other actin cytoskeletal proteins, e.g., the missing in metastasis (MIM) proteins (Mattila et al., 2003; Woodings et al., 2003). The espin WH2 domain appears functional in that it binds actin monomer in vitro and contributes to rapid actin monomer delivery to microvilli in transfected epithelial cells in vivo (Loomis et al., 2003). Actin monomer recruitment by espins may not be exclusively the purview of the $\mathrm{WH} 2$ domain; the proline-rich peptides of the espins, which are differentially represented among the espin isoforms, can bind profilin and, therefore, presumably also profilinactin monomer complex. Accordingly, the eight consecutive prolines in the $\mathrm{C}$-terminal proline-rich peptide of espin $2 \mathrm{~B}$ (Fig. 5D, PR2) also contribute to rapid actin monomer delivery to microvilli in vivo (Loomis et al., 2003). Thus, it may be relevant that espin 1 and the espin 2 isoforms contain two profilin-binding proline-rich peptides, the newly identified espin $3 \mathrm{~A}$ and espin $3 \mathrm{~B}$ contain one, and espin 4 contains none. By virtue of missing one of the two proline-rich peptides present in the larger espin isoforms (Fig. 5D, PR1), the espin 3 isoforms are unable to bind the SH3 domain of IRSp53. This difference might extend to other protein ligands with SH3 domains. Although recently reported to interact with actin directly (Yamagishi et al., 2004), IRSp53 could 
also potentially regulate espin 1 or the espin 2 isoforms downstream of small GTPases or associate with these espin isoforms in multiprotein complexes (Krugmann et al., 2001; Sekerková et al., 2003).

In view of the ability of all known espin isoforms to cause the elongation of microvillar PABs in vivo, we were surprised that espin $3 \mathrm{~A}$, espin $3 \mathrm{~B}$, and espin 4 proved to be such potent inhibitors of actin polymerization in vitro. This activity suggests a new way that espins could contribute to the regulation and dynamics of the actin cytoskeleton. The inhibitory effect is reminiscent of that observed for certain other proteins with functional WH2 domains, such as the MIM proteins (Mattila et al., 2003; Woodings et al., 2003). The basis for the inhibitory effect and the reason why it is not manifested by the larger espin isoforms, such as espin $2 \mathrm{~B}$, remain to be elucidated, but these observations raise the possibility that espin isoforms may also differentially affect other key parameters, such as the rate of nucleotide exchange on actin and assembly at the barbed versus the pointed end of actin filaments.

\section{Syndromic effects}

Finally, our demonstration that members of the espin family of actin cytoskeletal regulatory proteins are present in multiple sensory cell types raises the possibility that jerker (Zheng et al., 2000) and other espin mutations, such as those recently found in the DFNB36 families (Naz et al., 2004), lead to syndromic effects that are yet to be characterized in full. The findings of this study should prove useful in helping to pinpoint the entire roster of altered phenotypes in human espin mutants and in the design and interpretation of additional studies in animal models to analyze the roles of the different espin isoforms and the effects of espin mutations in different cell types. Our discovery that the different espin isoforms have distinct complements of binding sites and activities may ultimately help explain why some cells in espin mutants, e.g., hair cells, suffer different fates than other cells that normally express espins (Zheng et al., 2000).

\section{References}

Bartles JR (2000) Parallel actin bundles and their multiple actin-binding proteins. Curr Opin Cell Biol 12:72-78.

Bartles JR, Wierda A, Zheng L (1996) Identification and characterization of espin, an actin-binding protein localized to the F-actin-rich junctional plaques of Sertoli cell ectoplasmic specializations. J Cell Sci 109:1229-1239.

Bartles JR, Zheng L, Li A, Wierda A, Chen B (1998) Small espin: a third actin-bundling protein and potential forked protein ortholog in brush border microvilli. J Cell Biol 143:107-119.

Caicedo A, Pereira E, Margolskee RF, Roper SD (2003) Role of the G-protein subunit $\alpha$-gustducin in taste cell responses to bitter stimuli. J Neurosci 23:9947-9952.

Chen B, Li A, Wang D, Wang M, Zheng L, Bartles JR (1999) Espin contains an additional actin-binding site in its $\mathrm{N}$ terminus and is a major actinbundling protein of the Sertoli cell-spermatid ectoplasmic specialization junctional plaque. Mol Biol Cell 10:4327-4339.

Clapp TR, Stone LM, Margolskee RF, Kinnamon SC (2001) Immunocytochemical evidence for co-expression of Type III IP3 receptor with signaling components of bitter taste transduction. BMC Neurosci 2:6.

Clapp TR, Yang R, Stoick CL, Kinnamon SC, Kinnamon JC (2004) Morphologic characterization of rat taste receptor cells that express components of the phospholipase C signaling pathway. J Comp Neurol 468:311-321.

Cullen PJ, Cozier GE, Banting G, Mellor H (2001) Modular phosphoinositidebinding domains - their role in signaling and membrane trafficking. Curr Biol 11:R882-R893.

DeRosier DJ, Tilney LG (2000) F-actin bundles are derivatives of microvilli: what does this tell us about how bundles might form? J Cell Biol 148:1-6.

Dulac C, Torello AT (2003) Molecular detection of pheromone signals in mammals: from genes to behaviour. Nat Rev Neurosci 4:551-562.
Fieni F, Ghiaroni V, Tirindelli R, Pietra P, Bigiani A (2003) Apical and basal neurons isolated from the mouse vomeronasal organ differ for voltagedependent currents. J Physiol (Lond) 552:425-436.

Finger TE, Bottger B, Hansen A, Anderson KT, Alimohammadi H, Silver WL (2003) Solitary chemoreceptor cells in the nasal cavity serve as sentinels of respiration. Proc Natl Acad Sci USA 100:8981-8986.

Flanagan LA, Cunningham CC, Chen J, Prestwich GD, Kosik KS, Janmey PA (1997) The structure of divalent cation-induced aggregates of PIP2 and their alteration by gelsolin and tau. Biophys J 73:1440-1447.

Goldschmidt-Clermont PJ, Machesky LM, Baldassare JJ, Pollard TD (1990) The actin-binding protein profilin binds to PIP2 and inhibits its hydrolysis by phospholipase C. Science 247:1575-1578.

Halata Z, Grim M, Bauman KI (2003) Friederich Sigmund Merkel and his "Merkel cell," morphology, development and physiology: review and new results. Anat Rec 271A:225-239.

Hansel C, Linden DJ, D’Angelo E (2001) Beyond parallel fiber LTD: the diversity of synaptic and non-synaptic plasticity in the cerebellum. Nat Neurosci 4:467-475.

Higgs HN, Blanchoin L, Pollard TD (1999) Influence of the C terminus of Wiskott-Aldrich syndrome protein (WASp) and the Arp2/3 complex on actin polymerization. Biochemistry 38:15212-15222.

Holy TE, Dulac C, Meister M (2000) Responses of vomeronasal neurons to natural stimuli. Science 289:1569-1572.

Kaltenbach JA, Falzarano PR, Simpson TH (1994) Postnatal development of the hamster cochlea. II. Growth and differentiation of stereocilia bundles. J Comp Neurol 350:187-198.

Karolyi IJ, Probst FJ, Beyer L, Odeh H, Dootz G, Cha KB, Martin DM, Avraham KB, Kohrman D, Dolan DF, Raphael Y, Camper SA (2003) Myo15 function is distinct from Myo6, Myo7a and pirouette in development of cochlear stereocilia. Hum Mol Genet 12:2797-2805.

Krugmann S, Jordens I, Gevaert K, Driessens M, Vandekerchove J, Hall A (2001) Cdc42 induces filopodia by promoting the formation of an IRSp53:Mena complex. Curr Biol 11:1645-1655.

Lambrechts A, Braun A, Jonckheere V, Aszodi A, Lanier LM, Robbens J, Van Colen I, Vandekerckhove J, Fässler R, Ampe C (2000) Profilin II is alternatively spliced, resulting in profilin isoforms that are differentially expressed and have distinct biochemical properties. Mol Cell Biol 20:8209-8219.

Loomis PA, Zheng L, Sekerková G, Changyaleket B, Mugnaini E, Bartles JR (2003) Espin cross-links cause the elongation of microvillus-type parallel actin bundles in vivo. J Cell Biol 163:1045-1055.

Lucas P, Ukhanov K, Leinders-Zufall T, Zufall F (2003) A diacylglycerolgated cation channel in vomeronasal neurons is impaired in TRPC2 mutant mice: mechanism of pheromone transduction. Neuron 40:551-561.

Mattila PK, Salminen M, Yamashiro T, Lappalainen P (2003) Mouse MIM, a tissue-specific regulator of cytoskeletal dynamics, interacts with ATPactin monomers through its C-terminal WH2 domain. J Biol Chem 278:8452-8459.

Naz S, Griffith AJ, Riazuddin S, Hampton LL, Battey Jr JF, Khan SN, Riazuddin S, Wilcox ER, Friedman TB (2004) Mutations of ESPN cause autosomal recessive deafness and vestibular dysfunction. J Med Genet, in press.

Newman EA (2001) Propagation of intercellular calcium waves in retinal astrocytes and Müller cells. J Neurosci 21:2215-2223.

Petrella EC, Machesky LM, Kaiser DA, Pollard TD (1996) Structural requirements and thermodynamics of the interaction of proline peptides with profilin. Biochemistry 35:16535-16543.

Pickles JO, Corey DP (1992) Mechanoelectrical transduction by hair cells. Trends Neurosci 15:254-259.

Ricci AJ, Crawford AC, Fettiplace R (2003) Tonotopic variation in the conductance of the hair cell mechanotransducer channel. Neuron 40:983-990.

Rzadzinska AK, Schneider ME, Davies C, Riordan GP, Kachar B (2004) An actin molecular treadmill and myosins maintain stereocilia functional architecture and self-renewal. J Cell Biol 164:887-897.

Sechi AS, Wehland J (2000) The actin cytoskeleton and plasma membrane connection: PtdIns $(4,5) \mathrm{P}_{2}$ influences cytoskeletal protein activity at the plasma membrane. J Cell Sci 113:3685-3695.

Sekerková G, Loomis PA, Changyaleket B, Zheng L, Eytan R, Chen B, Mugnaini E, Bartles JR (2003) Novel espin actin-bundling proteins are localized to Purkinje cell dendritic spines and bind the Src homology 3 adapter protein insulin receptor substrate p53. J Neurosci 23:1310-1319. 
Surmeier DJ, Bargas J, Hemmings HC Jr, Naim AC, Greengard P (1995) Modulation of calcium currents by a D1 dopaminergic protein kinase/ phosphatase cascade in rat neostriatal neurons. Neuron 14:385-397.

Svitkina TM, Bulanova EA, Chaga OY, Vignjevic DM, Kojima S, Vasiliev JM, Borisy GG (2003) Mechanism of filopodia initiation by reorganization of a dendritic network. J Cell Biol 160:409-421.

Tachibana T, Endoh M, Kumakami R, Nawa T (2003) Immunohistochemical expressions of mGluR5, P2Y2 receptor, PLC-beta1, and IP3R-1 and -II in Merkel cells in rat sinus hair follicles. Histochem Cell Biol 120:13-21.

Taylor CW (2002) Controlling calcium entry. Cell 111:767-769.

Tilney LG, Connelly PS, Vranich KA, Shaw MK, Guild GM (1998) Why are two different cross-linkers necessary for actin bundle formation in vivo and what does each cross-linker contribute. J Cell Biol 143:121-133.

Wang J, Gambhir A, Hangyas-Mihalyne G, Murray D, Golebiewska U, McLaughlin S (2002) Lateral sequestration of phosphatidylinositol 4,5bisphosphate by the basic effector domain of myristoylated alanine-rich C kinase substrate is due to nonspecific electrostatic interactions. J Biol Chem 277:34401-34412.
Welch MD, Mullins RD (2002) Cellular control of actin nucleation. Annu Rev Cell Dev Biol 18:247-288.

Woodings JA, Sharp SJ, Machesky LM (2003) MIM-B, a putative metastasis suppressor protein, binds to actin and to protein tyrosine phosphatase $\delta$. Biochem J 371:463-471.

Yamagishi A, Masuda M, Ohki T, Onishi H, Mochizuki N (2004) A novel actin-bundling/filopodium-forming domain conserved in insulin receptor tyrosine kinase substrate $\mathrm{p} 53$ and missing in metastasis protein. J Biol Chem 279:14929-14936.

Yin HL, Janmey PA (2003) Phosphoinositide regulation of the actin cytoskeleton. Annu Rev Physiol 65:761-789.

Zancanaro C, Caretta CM, Merigo F, Cavaggioni A, Osculati F (1999) alpha-Gustducin expression in the vomeronasal organ of the mouse. Eur J Neurosci 11:4473-4475.

Zheng L, Sekerková G, Vranich K, Tilney LG, Mugnaini E, Bartles JR (2000) The deaf jerker mouse has a mutation in the gene encoding the espin actinbundling proteins of hair cell stereocilia and lacks espins. Cell 102:377-385.

Zufall F, Kelliher KR, Leinders-Zufall T (2002) Pheromone detection by mammalian vomeronasal neurons. Microsc Res Tech 58:251-260. 